\title{
Clocking Femtosecond X Rays
}

A. L. Cavalieri, ${ }^{1}$ D. M. Fritz, ${ }^{1}$ S. H. Lee,${ }^{1}$ P. H. Bucksbaum, ${ }^{1}$ D. A. Reis, ${ }^{1}$ J. Rudati, ${ }^{2}$ D. M. Mills, ${ }^{2}$ P. H. Fuoss,${ }^{3}$ G. B. Stephenson, ${ }^{3}$ C. C. Kao, ${ }^{4}$ D. P. Siddons,${ }^{4}$ D. P. Lowney, ${ }^{5}$ A. G. MacPhee, ${ }^{5}$ D. Weinstein, ${ }^{5}$ R. W. Falcone, ${ }^{5}$ R. Pahl, ${ }^{6}$ J. Als-Nielsen, ${ }^{7}$ C. Blome, ${ }^{8}$ S. Düsterer, ${ }^{8}$ R. Ischebeck, ${ }^{8}$ H. Schlarb, ${ }^{8}$ H. Schulte-Schrepping, ${ }^{8}$ Th. Tschentscher, ${ }^{8}$ J. Schneider, ${ }^{8}$ O. Hignette, ${ }^{9}$ F. Sette, ${ }^{9}$ K. Sokolowski-Tinten,${ }^{10}$ H. N. Chapman, ${ }^{11}$ R. W. Lee, ${ }^{11}$ T. N. Hansen, ${ }^{12}$ O. Synnergren, ${ }^{12}$ J. Larsson, ${ }^{12}$ S. Techert, ${ }^{13}$ J. Sheppard,${ }^{14}$ J. S. Wark,${ }^{14}$ M. Bergh, ${ }^{15}$ C. Caleman, ${ }^{15}$ G. Huldt, ${ }^{15}$ D. van der Spoel,${ }^{15}$ N. Timneanu, ${ }^{15}$ J. Hajdu,${ }^{15}$ R. A. Akre, ${ }^{16}$ E. Bong, ${ }^{16}$ P. Emma,${ }^{16}$ P. Krejcik, ${ }^{16}$ J. Arthur, ${ }^{17}$ S. Brennan, ${ }^{17}$ K. J. Gaffney, ${ }^{17}$ A. M. Lindenberg, ${ }^{17}$ K. Luening, ${ }^{17}$ and J. B. Hastings ${ }^{17}$

${ }^{1}$ FOCUS Center, Departments of Physics and Applied Physics Program, University of Michigan, Ann Arbor, MI 48109, USA

${ }^{2}$ Advanced Photon Source, Argonne National Laboratory, Argonne, IL 60439, USA

${ }^{3}$ Materials Science Division, Argonne National Laboratory, Argonne, IL 60439, USA

${ }^{4}$ National Synchrotron Light Source, Brookhaven National Laboratory, Upton, NY 11973, USA

${ }^{5}$ Department of Physics, University of California, Berkeley, CA 94720, USA

${ }^{6}$ Consortium for Advanced Radiation Sources, The University of Chicago, Chicago, IL 60637, USA

${ }^{7}$ Niels Bohr Institute, Copenhagen University, 2100 Copenhagen $\emptyset$, Denmark

${ }^{8}$ Deutsches Elektronen-Synchrotron DESY, Notkestrasse 85, 22607 Hamburg, Germany

${ }^{9}$ European Synchrotron Radiation Facility, 38043 Grenoble Cedex 9, France

${ }^{10}$ Institut für Optik und Quantenelektronik, Friedrich-Schiller-Universität Jena, Max-Wien-Platz 1, 07743 Jena, Germany

${ }^{11}$ Physics Department, Lawrence Livermore National Laboratory, Livermore, CA 94550, USA

${ }^{12}$ Department of Physics, Lund Institute of Technology, P.O. Box 118, S-22100, Lund, Sweden

${ }^{13}$ Max Plank Institute for Biophysical Chemistry, Am Faßberg 11, 37077 Göttingen, Germany

${ }^{14}$ Department of Physics, Clarendon Laboratory, Parks Road, University of Oxford, Oxford OX1, 3PU, United Kingdom

${ }^{15}$ Department of Cell and Molecular Biology, Biomedical Centre, Uppsala University, SE-75124 Uppsala, Sweden

${ }^{16}$ Stanford Linear Accelerator Center, Menlo Park, CA 94025, USA

${ }^{17}$ Stanford Synchrotron Radiation Laboratory/SLAC, Menlo Park, CA 94025, USA

(Received 2 November 2004; published 24 March 2005)

Linear-accelerator-based sources will revolutionize ultrafast $x$-ray science due to their unprecedented brightness and short pulse duration. However, time-resolved studies at the resolution of the X-ray pulse duration are hampered by the inability to precisely synchronize an external laser to the accelerator. At the Sub-Picosecond Pulse Source at the Stanford Linear-Accelerator Center we solved this problem by measuring the arrival time of each high energy electron bunch with electro-optic sampling. This measurement indirectly determined the arrival time of each x-ray pulse relative to an external pump laser pulse with a time resolution of better than $60 \mathrm{fs}$ rms.

DOI: 10.1103/PhysRevLett.94.114801

PACS numbers: 41.60.Cr, 41.75.Ht, 42.65.Re

Ultrafast x-ray pulses are providing our first view of subpicosecond atomic motion. New sources based on high harmonic generation [1,2] and laser-produced plasmas [3] as well as femtosecond laser-sliced synchrotron emission [4] have been demonstrated. These sources produce $\mathrm{x}$-ray pulses with durations of less than a few hundred femtoseconds, the time scale of vibrations in solids and molecules and the making and breaking of chemical bonds. While these sources provide the time resolution necessary to study these dynamics, their relatively low brightness limits their application and often hinders attempted experiments.

A new generation of linear-accelerator-based $\mathrm{x}$-ray free electron lasers (XFELs) will be more than 20 orders of magnitude brighter than laser-plasma-based sources and have the potential to produce $\mathrm{x}$-ray pulses below one femtosecond in duration [5]. With $\mathrm{x}$ rays from an XFEL, researchers can expect to image chemistry in real time on the atomic scale. While these new XFELs will be far brighter than any other ultrafast X-ray source, their physical size and complexity introduce new challenges which, if left unaddressed, will restrict their application. A major obstacle will be the inability to precisely synchronize the time-dependent process being studied with the $\mathrm{x}$-ray pulse generated by a large accelerator-based source.

Subpicosecond time-dependent phenomena are typically studied with pump-probe techniques in which the dynamics are initiated by an ultrafast laser or laser-driven source and then probed after a time delay. If these experiments can be self-synchronized, with the pump and probe having a common laser source, then precise time delays can be produced using different optical path lengths. The time resolution is then limited by the overlap of the pump and probe pulses that can be as short as a fraction of a 
femtosecond [6]. An XFEL source will usually provide only one pulse, either pump or probe. Since timing jitter between the pump and probe will degrade temporal resolution, the level of synchronization between the XFEL and an external ultrafast laser is a primary concern. The SubPicosecond Pulse Source (SPPS) at the Stanford LinearAccelerator Center (SLAC) is a linac-based x-ray source with synchronization challenges similar to those that will confront XFEL sources. The electron acceleration and bunch compression schemes used for SPPS and XFELs are nearly identical. SPPS is the first source to employ compressed femtosecond electron bunches to produce femtosecond $\mathrm{x}$-ray pulses $[7,8]$ and is currently the world's brightest ultrafast $\mathrm{x}$-ray source.

Synchronization between a linac-based source and an external laser to within a few picoseconds can be achieved for short periods of time if the external laser repetition rate is tuned to a submultiple of the linac radio frequency (rf). Pulse-to-pulse variation in electron bunch charge and linac rf noise, however, introduce variations in bunch arrival time which effectively prohibit absolute synchronization at the subpicosecond level. Rather than attempting to improve the synchronization of the external laser to the accelerator we solved the problem by indirectly measuring the arrival time of each individual $\mathrm{x}$-ray pulse with respect to a pump laser pulse. Although the time of arrival fluctuates from pulse to pulse, the information can be used to place measurements in sequence with precise time resolution. The time resolution of experiments performed at SPPS then approaches the fundamental limit fixed by the $\mathrm{X}$-ray pulse duration.

The pulse-by-pulse timing information is obtained using a noninvasive technique based on electro-optic sampling (EOS) of the electric field surrounding the ultrarelativistic electron bunch that produces the x-ray pulse. Bunches of up to $2 \times 10^{10}$ electrons are precompressed, accelerated through the $3 \mathrm{~km}$ SLAC linear accelerator to an ultrarelativistic energy of $E=28.5 \mathrm{GeV}$, and then compressed once more before delivery to SPPS. Electron trajectory simulations show that the electron bunch length at SPPS is $10 \mu \mathrm{m} \mathrm{rms}$ along the direction of motion, corresponding to $80 \mathrm{fs}$ FWHM. As a consequence of special relativity, the Coulomb field of the electron bunch is nearly transverse to its motion. Its magnitude is the vector sum of the fields of the individual electrons. We estimate a peak electric field of hundreds of megavolts per meter at a few millimeters from the beam.

If a crystal is placed adjacent to the electron beam, its index of refraction will be distorted anisotropically by the strong electromagnetic fields associated with the ultrarelativistic electrons $[9,10]$. This transient birefringence or electro-optic effect is induced without affecting the electron bunch propagation. Laser light passing through the crystal at the same time as the transient birefringence will have its polarization rotated and can be used to probe the effect [11]. In the limit that the crystal response is elec- tronic, this transient birefringence is prompt, and the effect is proportional to the electron bunch current profile.

Detection of the electro-optic signal that is imprinted on the probe laser polarization is challenging because the electron bunch is expected to be shorter than $100 \mathrm{fs}$. However, if an ultrafast laser pulse is swept across the EO crystal at an angle to the electron beam trajectory, time is mapped into space [12]. As shown in Fig. 1, the top edge of the laser pulse intersects the crystal before the bottom edge so that a different cross section of the laser pulse interacts with the electron beam depending on its time of arrival. The angle of incidence of the laser probe determines the sweep rate, and consequently the resolution of the measurement, while the laser beam diameter determines the duration of the measurement window. A polarizer transmits only the portion of the probe laser where the polarization was altered by the EO effect, producing a time-dependent image of the electron bunch. The position of the signal indicates the bunch's time of arrival, while its width and amplitude contain information on the temporal current profile.

The time resolution of the measurement is limited by the thickness of the electro-optic crystal [13], the pulse length of the laser, and the spatial resolution of the imaging system used to monitor the transmitted laser pulse. For the measurements presented here we used a $200 \mu \mathrm{m}$ thick $\langle 110\rangle \mathrm{ZnTe}$ crystal positioned $5 \mathrm{~mm}$ from the electron
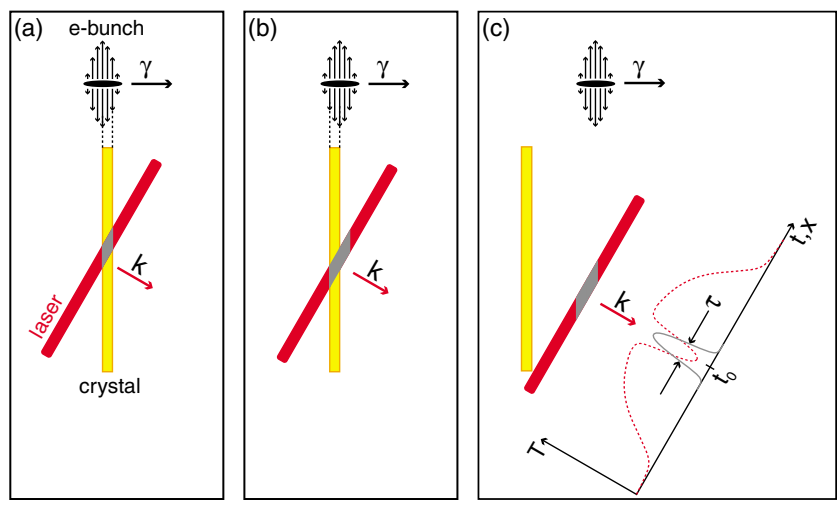

FIG. 1 (color). Cartoon depiction of spatially resolved electrooptic sampling. The frames represent three instants during the course of the measurement. The yellow object represents the EO crystal. The red object represents an ultrafast laser probe pulse moving from top left to bottom right. The black oval represents the ultrarelativistic electron bunch moving from left to right (with electric field lines indicated). In (a), the front of the electron bunch and the laser pulse interact in the EO crystal. In (b), the back of the electron bunch interacts with the laser pulse. In (c) the electron bunch has passed the crystal and its shape has been imprinted on the laser polarization profile, mapping time into space. The intensity profiles of the laser polarization components are plotted. The width of the signal, $\tau$, represents a convolution of the electron bunch length, the crystal EO response, and the laser pulse duration. The centroid of the signal indicates the relative time of arrival. 
bunch trajectory. The electric field due to the bunch lies in the plane of the crystal. The $2 \mathrm{~mm}$ wide laser beam is incident at an angle of $45^{\circ}$ with respect to the crystal surface normal with an effective single-shot window of 9 ps.

The laser oscillator operates at an actively-stabilized repetition rate of $102 \mathrm{MHz}$, a subharmonic of the linearaccelerator rf signal at SLAC, and is phase-locked to this reference to better than $200 \mathrm{fs}$ (rms integrated phase jitter from $1 \mathrm{~Hz}$ to $40 \mathrm{MHz}$ ); EOS can be used to measure and compensate for the jitter and drift existing between the reference $r f$ and the electron bunch arrival time. To achieve precise timing, the EOS measurement and x-ray pumpprobe experiments share a common laser system. A single Ti:sapphire oscillator is located in a laboratory next to the end of the x-ray beam line, approximately $150 \mathrm{~m}$ from the EOS experiment. This large separation presents a unique challenge for laser transport and timing, which will be characteristic of future XFEL facilities.

Ultrafast optical pulses from the oscillator are delivered to the EO crystal via a polarization-preserving single-mode optical fiber. Short optical pulses are required for EOS; however, ultrafast pulses broaden rapidly upon propagation in an optical fiber. For example, a 100 fs optical pulse will double its length after traveling only a few centimeters due to spectral dispersion in the glass fiber. We overcame this material dispersion by first spectrally dispersing the oscillator pulses using a series of gratings and a programmable spectral phase mask [14]. The required spectral phase shifts are programmed into the phase mask by a genetic learning algorithm [15]. The dispersion is opposite to that of the fiber, so that the pulses are recompressed as they travel through the fiber to the EOS experiment. Final compression occurs in glass optical elements just before the pulses encounter the EO crystal. In our experiments we delivered a 135 fs FWHM laser pulse to the EO crystal.

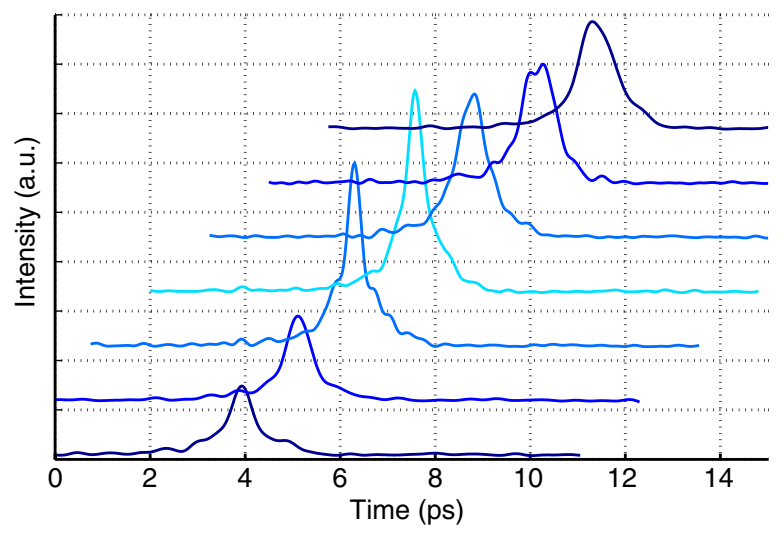

FIG. 2 (color online). A series of electro-optic signals (offset for display) collected as the electron bunch compressors at SLAC were changed, changing the bunch length. The minimum FWHM shown represents a time of $270 \mathrm{fs}$ which is the resolution limit of the apparatus.
A series of EOS images of the electron bunch, taken as a function of bunch compression, are shown in Fig. 2. These are the shortest single-shot electron bunch measurements to date [16]. The width of the EO signal reaches a minimum of 270 fs FWHM, which is a convolution of the instrument response and the electron bunch duration, and appears to be instrument resolution limited. These data show the optimum compression settings and indicate that EOS could also be used as an electron bunch length diagnostic at future XFELs. A combination of a thinner crystal, a shorter laser pulse, and better imaging would be required to resolve the predicted $80 \mathrm{fs}$ bunch duration at SPPS.

Twenty consecutive single-shot measurements made at SPPS are shown in Fig. 3. The centroid of each EO image can be determined with 30 fs accuracy, and indicates the time of arrival of the bunch with respect to the laser pulse. Random fluctuations from shot to shot are evident and were measured to be $194 \mathrm{fs}$ rms over $100 \mathrm{~s}$ of observation.

The laser fiber transport system can introduce additional sources of jitter between the EOS measurement and the $\mathrm{x}$-ray pulse time of arrival at an SPPS x-ray-pump-probe experiment. In order to determine the magnitude of this additional jitter we compared timing information obtained using EOS with that from a laser-pump-x-ray-probe study of ultrafast nonthermal melting of an InSb crystal [17]. A strong subpicosecond reduction in Bragg diffraction following intense laser excitation allowed for single-shot measurement of the relative arrival time of the $\mathrm{x}$-ray pulse and pump laser pulse $[18,19]$. This independent and direct measurement of the relative $\mathrm{x}$-ray timing was made in the $\mathrm{x}$-ray experiment hutch and could be determined to within $50 \mathrm{fs}$. Simultaneous X-ray and EOS measurements were collected over $30 \mathrm{~s}$. The comparison is shown in Fig. 4. While each timing measurement method individually yields a jitter of approximately $200 \mathrm{fs}$, the correlation shows only $60 \mathrm{fs}$ rms jitter between the two techniques.
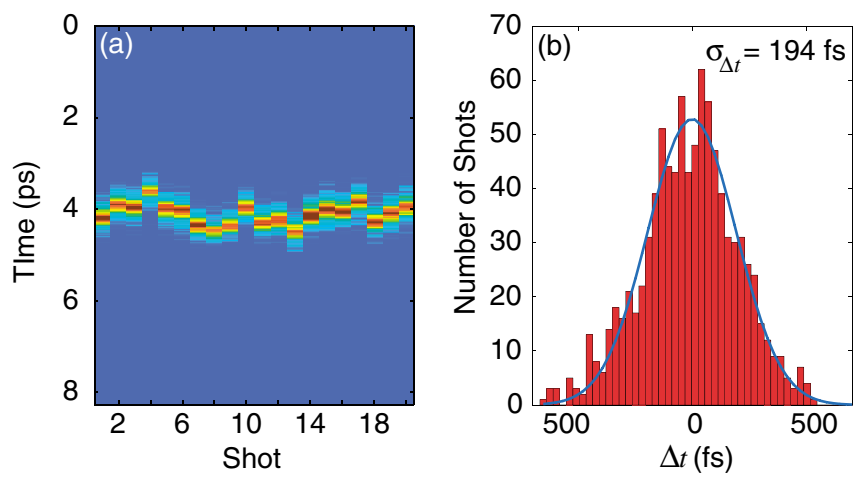

FIG. 3 (color). (a) Twenty consecutive single-shot electron bunch measurements. The bright band in each column is the electro-optic signal, its location indicates the time of arrival of the electron bunch with respect to the laser probe pulse, and its width corresponds to the electron bunch duration. (b) Normalized arrival time histogram of 1000 consecutive single shots. 

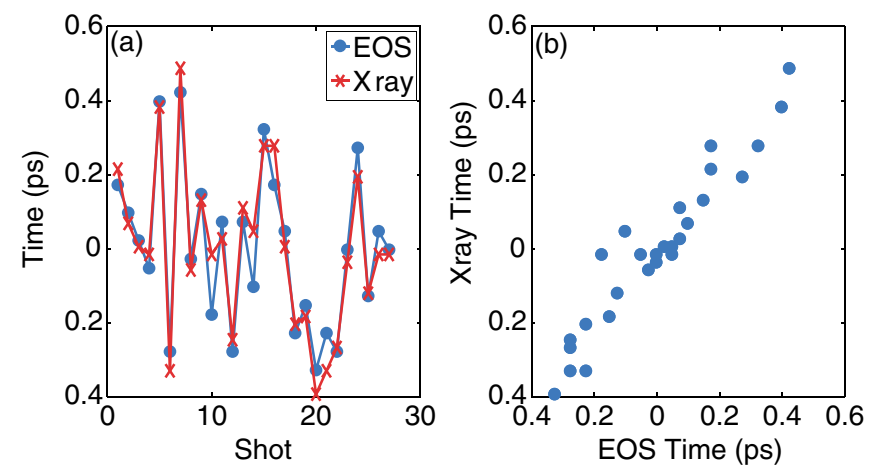

FIG. 4 (color). (a) Shot-by-shot comparison of pulse arrival times determined from EOS measurements (blue), and from ultrafast laser-pump-x-ray-probe measurements (red). Both measurement techniques show a shot-to-shot jitter of $200 \mathrm{fs}$. (b) Correlation plot of the data shown in (a). The relative jitter between the two techniques is $60 \mathrm{fs}$.

The short-term timing jitter at SPPS is approximately $200 \mathrm{fs}$. There are also long-term drifts, associated with the rf reference to which the laser oscillator is phase-locked and with the fiber laser transport system. Most pump-probe experiments, which require accumulation of data over multiple shots, would have their time resolution limited by these long-term drifts, which can be as great as $30 \mathrm{ps}$ at SPPS. Electron bunch measurements made using EOS could be used to identify and compensate for the sources of drift. Therefore, a continuous set of indirect x-ray pulse measurements can be collected and used to improve the time resolution of an SPPS x-ray pump-probe experiment from $30 \mathrm{ps}$ to $60 \mathrm{fs}$ or less.

Ultrafast research at linear-accelerator-based sources requires knowledge of the x-ray pulse arrival time with respect to a pump laser with femtosecond precision. This Letter introduces the first technique that specifically addresses this problem. By measuring the electron bunch timing, sub-100 fs precision in the x-ray arrival time was demonstrated. The time resolution for experiments at SPPS has been improved to $60 \mathrm{fs}$ rms, nearing the resolution limit imposed by the $\mathrm{x}$-ray pulse duration. Relative timing information from spatially resolved EO measurements could probably be extended to $5 \mathrm{fs}$, matching the projected performance of XFELs into the foreseeable future

Portions of this research were supported by the U.S. Department of Energy, Office of Basic Energy Science through direct support for the SPPS and individual investigators and through the Stanford Synchrotron Radiation Laboratory, a national user facility operated by Stanford University. Additional support for the construction of SPPS was provided by Uppsala University through a grant from the Swedish Research Council.
[1] C. Spielmann, N. H. Burnett, S. Sartania, R. Koppitsch, M. Schnurer, C. Kan, M. Lenzner, P. Wobrauschek, and F. Krausz, Science 278, 661 (1997).

[2] Z. H. Chang, A. Rundquist, H. W. Wang, M. M. Murnane, and H. C. Kapteyn, Phys. Rev. Lett. 79, 2967 (1997).

[3] C. Rischel, A. Rousse, I. Uschmann, P. A. Albouy, J. P. Geindre, P. Audebert, J.C. Gauthier, E. Forster, J. L. Martin, and A. Antonetti, Nature (London) 390, 490 (1997).

[4] R.W. Schoenlein, S. Chattopadhyay, H. H. W. Chong, T. E. Glover, P. A. Heimann, C. V. Shank, A. A. Zholents, and M.S. Zolotorev, Science 287, 2237 (2000).

[5] R. F. Service, Science 298, 1356 (2002).

[6] M. Hentschel, R. Kienberger, C. Spielmann, G. A. Reider, N. Milosevic, T. Brabec, P. Corkum, U. Heinzmann, M. Drescher, and F. Krausz, Nature (London) 414, 509 (2001).

[7] P. Emma, R. Iverson, P. Krejcik, P. Raimondi, and J. Safrarek, in Particle Accelerator Conference, Chicago, Illinois, edited by $\mathrm{P}$. Lucas and S. Webber (IEEE, New York, 2001).

[8] L. Bentson, P. Emma, and P. Krejcik, in European Particle Accelerator Conference, Paris, France, 2002, edited by T. Garvey, J. Le Duff, P. Le Roux, Ch. Petit-Jean-Genaz, J. Poole, and L. Rivkin (CERN, Geneva, 2002), http:// accelconf.web.cern.ch/AccelConf/e02/default.htm.

[9] M.J. Fitch, A.C. Melissinos, P. L. Colestock, J.P. Carneiro, H. T. Edwards, and W. H. Hartung, Phys. Rev. Lett. 87, 034801 (2001).

[10] I. Wilke, A. M. MacLeod, W. A. Gillespie, G. Berden, G. M. H. Knippels, and A. F. G. van der Meer, Phys. Rev. Lett. 88, 124801 (2002)

[11] J.A. Valdmanis and G. Mourou, IEEE J. Quantum Electron. 22, 69 (1986).

[12] J. Shan, A. S. Weling, E. Knoesel, L. Bartels, M. Bonn, A. Nahata, G. A. Reider, and T. F. Heinz, Opt. Lett. 25, 426 (2000).

[13] Q. Wu and X.C. Zhang, Appl. Phys. Lett. 68, 1604 (1996).

[14] S. H. Lee, A. L. Cavalieri, D. M. Fritz, M. Myaing, and D. A. Reis, Opt. Lett. 29, 2602 (2004).

[15] T. Baumert, T. Brixner, V. Syfried, M. Strehle, and G. Gerber, Appl. Phys. B 65, 779 (1997).

[16] G. Berden, S. P. Jamison, A. M. MacLeod, W. A. Gillespie, B. Redlich, and A. F. G. van der Meer, Phys. Rev. Lett. 93, 114802 (2004).

[17] A. Rousse, C. Rischel, S. Fourmaux, I. Uschmann, S. Sebban, G. Grillon, P. Balcou, E. Foster, J. P. Geindre, P. Audebert et al., Nature (London) 410, 65 (2001).

[18] A. M. Lindenberg, J. Larsson, K. Sokolowski-Tinten, K. Gaffney, C. Blome, O. Synnergren, J. Sheppard, C. Caleman, A.G. MacPhee, D. Weinstein et al., Science (to be published).

[19] O. Synnergren, M. Harbst, T. Missalla, J. Larsson, G. Katona, R. Neutze, and R. Wouts, Appl. Phys. Lett. 80, 3727 (2002). 